\title{
A Population Genetic Model of Evolution of Host-Mate Attraction and Nonhost Repulsion in a Bark Beetle Pityogenes bidentatus
}

\author{
John A. Byers \\ US Arid-Land Agricultural Research Center, USDA-ARS, 21881 North Cardon Lane, Maricopa, AZ 85138, USA \\ Correspondence should be addressed to John A. Byers, john.byers@ars.usda.gov \\ Received 12 December 2011; Accepted 14 January 2012 \\ Academic Editor: Qing-He Zhang \\ Copyright () 2012 John A. Byers. This is an open access article distributed under the Creative Commons Attribution License, which \\ permits unrestricted use, distribution, and reproduction in any medium, provided the original work is properly cited. \\ Studies have shown that the bark beetle Pityogenes bidentatus (Coleoptera, Curculionidae, Scolytinae) avoids volatiles of nonhost \\ trees (Norway Spruce, birch, and oak) and healthy host Scotch Pine when orienting to aggregation pheromone. A population \\ genetic model of two behavioral genes was hypothesized where AA, Aa, and aa were allele combinations regulating orientation to \\ host tree and pheromone odors, and $\mathrm{BB}, \mathrm{Bb}$, and bb were combinations allowing avoidance of nonhost and unsuitable host odors. \\ The nine possible genotypes were assigned different survival factors that remained constant during simulation. The initial propor- \\ tion of aabb genotype (little aggregation/host response and little avoidance of nonhosts) was $\sim 1.0$ when a mutation was hypothe- \\ sized that caused better orientation to host/beetle odors (Aabb) and another mutation causing more efficient avoidance of nonhosts \\ $(\mathrm{aaBb})$. After these initial mutations, the model used indiscriminate mating of genotypic proportions and subsequent survival \\ as input for each successive generation. The results indicate that AABB eventually fixates in the populations in some scenarios, \\ while $\mathrm{AABB}$ and other genotypes reach stable equilibriums in other models depending on genotypic survival values supported by \\ ecologically sound assumptions. The models indicate how development of insecticide resistance in pest insects may proceed.
}

\section{Introduction}

Individuals of Pityogenes bidentatus (Herbst) (Coleoptera, Scolytinae) are fairly small ( $2-3 \mathrm{~mm}$ long) bark beetles that only colonize Scotch Pine, Pinus sylvestris L. during a yearly mating flight that occurs in April-May depending on the latitude [1]. These beetles are common in mixed deciduous and conifer forests of northern Europe where they prefer to feed on smaller diameter trunks and limbs of weakened hosts [1]. By 1989, the beetle had become established in the northeastern United States [2]. The males in flight appear to find weakened hosts by a combination of attraction to aggregation pheromone, (S)-cis-verbenol and grandisol [3-7], and by avoiding odors from nonhost deciduous trees [such as birch, Betula pendula Roth; Rowan (mountain ash), Sorbus aucuparia L.; English oak, Quercus robur L.; alder buckthorn (glossy buckthorn), Frangula alnus Mill.], and conifers Norway Spruce, Picea abies L., and fresh/healthy host Scotch Pine $[5,6]$. In these studies, aggregation components, $(S)$ cis-verbenol and grandisol, were placed inside each of a pair of barrier traps separated $6 \mathrm{~m}$ apart at $1.2 \mathrm{~m}$ height and revolved slowly at $2 \mathrm{rph}$ to even out any trap position effects [5-8]. One of the traps in the pair also had an inhibitory source, either monoterpenes $(1 \mathrm{mg} / \mathrm{h})$ or other synthetic plant volatiles, or a fine screen cage containing freshly cut bark chips or twigs with leaves/needles (80 to $200 \mathrm{~g}$ ) of hosts or nonhosts.

Odors from unsuitable hosts and nonhosts have not been tested alone without aggregation pheromone, so it is not certain that the plant odors can act alone during the beetle's dispersal and search for hosts. However, it was observed that the plant volatiles did repel the beetles in flight as they approached to within $1 \mathrm{~m}$ from a source of aggregation pheromone [5]. Many individual monoterpenes and blends released at rates comparable to that released from physical wounds of trees also inhibited attraction to their aggregation pheromone [5-7]. Earlier, a body of evidence had accumulated that attraction responses of conifer-infesting bark beetles in several genera are reduced by volatiles from nonhost angiosperm trees (e.g., Betula, Populus, Acer) [9-20]. Conifers such as pines and spruce usually produce resin, consisting of about $80 \%$ of mildly toxic monoterpenes, in order to 
defend against the penetrations of the attacking bark beetles $[21,22]$.

Once a male finds suitable host pine bark, he releases an aggregation pheromone that probably assists most individuals in finding suitable host and breeding habitat [23, 24]. The avoidance of nonhost volatiles may aid the pioneer males in finding suitable hosts during extensive searches as well as aid individuals while landing on colonized bark to avoid nearby nonhosts. On the other hand, little or nothing is known about the behavioral responses needed to select the appropriate host substrate, but it can be hypothesized that there is some attraction to host volatiles that might occur at close range after landing. Interestingly, host pine monoterpenes were only repellent to $P$. bidentatus during flight when responding to aggregation pheromone [5-7] and not when walking (Byers unpublished). Bolts cut from standing Scotch Pine placed in the forest were not colonized by P. bidentatus for several weeks during the same time that the beetles were caught in the hundreds on pheromone-baited traps (personal observations). However, several weeks later these bolts became infested, suggesting either a random landing after avoiding nonhosts or a weak attraction to fermenting host volatiles. Various monoterpene blends could indicate to arriving beetles that the trunk was the appropriate host since different tree species have different sets of monoterpenes [25, 26]. In a few cases, bark beetles in the genus Tomicus are significantly attracted to Scotch Pine and to its monoterpenes, especially $\alpha$-pinene (both enantiomers), 3 -carene, and terpinolene [27-30]. Several studies have found that certain monoterpenes enhance the attraction to pheromone components in some of the more "aggressive" bark beetles that kill standing trees [31-34].

The objective was to construct a population genetic computer model of evolution with selection of hypothetical genotypes of P. bidentatus with two genes each with two alleles, one gene for attraction to host/beetle semiochemicals (A and a) and the second for repulsion by nonhost semiochemicals ( $\mathrm{B}$ and $\mathrm{b}$ ). This means that there would be nine possible genotypes conferring special survival or reproductive benefits for each genotype that remained constant throughout the simulation of a specified number of generations. Throughout the population and in every generation, mating was assumed indiscriminate and proportional to each genotype currently present $[35,36]$. The nine-by-nine pairings of genotypes gives 81 possible pairings resulting in certain proportions of the nine genotypes, each generation based on the preceding population's proportions of each genotype. The initial proportion of aabb (little or no repulsion by nonhosts and little attraction to hosts) was the prevailing genotype except that one individual would have a mutation of a $\rightarrow \mathrm{A}$, and a second individual would have a mutation of $\mathrm{b} \rightarrow \mathrm{B}$ to begin the simulations. At each generation the proportions of each genotype were calculated and used as input for the next generation. Survival factors were set initially for the nine genotypes based on logical assumptions. For example, genotype aabb would have a low survival compared to AABB since the latter's individuals would avoid toxic nonhosts $(\mathrm{BB})$ and be attracted to hosts (AA); heterozygous (Aa or $\mathrm{Bb}$ ) would be intermediate in survival. The results of the models following an evolutionary mutational event would reveal the dramatic to gradual genotypic changes that might be expected during a number of generations resulting in gene fixation or gene equilibrium depending on the survival benefits of the mutated alleles. The same processes illustrated by the models help in understanding the population dynamics of pest insects that overcome crop plant resistance or develop resistance to insecticides [36-38].

\section{Materials and Methods}

A genetic model of evolution with two alleles, A and a, for attraction to hosts and two alleles, B and b, for repulsion from nonhosts was developed. This two-gene model has nine possible combinations of alleles AABB, AABb, AAbb, AaBB, $\mathrm{AaBb}, \mathrm{Aabb}, \mathrm{aaBB}, \mathrm{aaBb}$, and aabb that can be found in male and female beetles. Mating proceeds according to the proportion of each genotype (pan mixing) giving 81 possible pairings as shown in Table 1 . However, the genotype offspring in the table's boxes in the lower left of the diagonal line of the outlined boxes are replicated in the upper diagonal half. Thus, the number of unique pairings is reduced to $9+$ $32 / 2=45$ as shown in Algorithm 1. This algorithm takes the proportion $\left(\mathrm{P}_{1}\right.$ to $\left.\mathrm{P}_{9}\right)$ of each of the male and female mated genotypes (progeny are equally female and male) and multiplies it by the indicated proportions $(1,2,4,8$, or 16$)$ times 2 for those not in the diagonal line of the boxes or as indicated if in the diagonal line. Each such value is multiplied by the survival factor $\left(S_{1}\right.$ to $\left.S_{9}\right)$ for the appropriate genotype. For example, reasonable survival factors that are relative to each other might be $S_{1}=1$ for AABB, $S_{2}=0.9$ for AABb, $S_{3}=$ 0.5 for AAbb, $S_{4}=0.8$ for AaBb, $S_{5}=0.6$ for AaBb, $S_{6}=0.3$ for Aabb, $S_{7}=0.4$ for aaBB, $S_{8}=0.3$ for aaBb, and $S_{9}=0.2$ for aabb. These survival factors can just as well be any values as long as they are relative in magnitude (e.g., 10, 9, 5, 8, 6, 3, 4, 3 , and 2) since the sums of all multiplications for each of the nine genotypes $\left(G_{1}\right.$ to $\left.G_{9}\right)$ are then expressed as a proportion of the total sum of the nine genotypes $\left(\mathrm{P}_{1}\right.$ to $\left.\mathrm{P}_{9}\right)$ according to the following:

$$
\mathrm{P}_{1}=\frac{\mathrm{G}_{1}}{\sum_{k=1}^{9} \mathrm{G}_{k}} .
$$

The updated $\mathrm{P}_{1}$ to $\mathrm{P}_{9}$ values then serve as the mating proportions of the genotypes for the next generation, iterating until the last generation is attained to obtain the ending genotypic frequencies.

The initial population number based on the initial frequencies would be $10^{7}$. The population would have an initial proportion of almost all aabb $\left(\mathrm{P}_{9}=0.9999998\right)$, except one individual would mutate to Aabb $\left(\mathrm{P}_{6}=0.0000001\right)$ and another would mutate to aaBb $\left(\mathrm{P}_{8}=0.0000001\right)$, and then the model would proceed as described above for at least 100 generations. The model can accommodate any population size by adjusting the initial proportions of the genotypes. The survival factor of each genotype determines the ultimate proportion of each genotype, and as such the possibilities appear unlimited. However, the relative survival of the nine genotypes is constrained as will be evident in four examples 
TABLE 1: Nine genotypes of each sex and the 81 possible crossings and their proportions.

\begin{tabular}{|c|c|c|c|c|c|c|c|c|c|}
\hline & $\mathrm{AABB}$ & $\mathrm{AABb}$ & $\mathrm{AAbb}$ & AaBB & $\mathrm{AaBb}$ & Aabb & $\mathrm{aaBB}$ & $\mathrm{aaBb}$ & aabb \\
\hline $\mathrm{AABB}^{1}$ & 16АABB & $\begin{array}{l}8 \mathrm{AABB} \\
8 \mathrm{AABb}\end{array}$ & $16 \mathrm{AABb}$ & $\begin{array}{l}8 \mathrm{AABB} \\
8 \mathrm{AaBB}\end{array}$ & $\begin{array}{l}4 \mathrm{AABB} \\
4 \mathrm{AABb} \\
4 \mathrm{AaBB} \\
4 \mathrm{AaBb}\end{array}$ & $\begin{array}{l}8 \mathrm{AABb} \\
8 \mathrm{AaBb}\end{array}$ & $16 \mathrm{AaBB}$ & $\begin{array}{l}8 \mathrm{AaBB} \\
8 \mathrm{AaBb}\end{array}$ & $16 \mathrm{AaBb}$ \\
\hline $\mathrm{AABb}$ & $\begin{array}{l}8 \mathrm{AABB} \\
8 \mathrm{AABb}\end{array}$ & $\begin{array}{l}4 \mathrm{AABB} \\
8 \mathrm{AABb} \\
4 \mathrm{AAbb}\end{array}$ & $\begin{array}{l}8 \mathrm{AABb} \\
8 \mathrm{AAbb}\end{array}$ & $\begin{array}{l}4 \mathrm{AABB} \\
4 \mathrm{AABb} \\
4 \mathrm{AaBB} \\
4 \mathrm{AaBb}\end{array}$ & $\begin{array}{l}2 \mathrm{AABB} \\
4 \mathrm{AABb} \\
2 \mathrm{AAbb} \\
2 \mathrm{AaBB} \\
4 \mathrm{AaBb} \\
2 \mathrm{Aabb}\end{array}$ & $\begin{array}{l}4 \mathrm{AABb} \\
4 \mathrm{AAbb} \\
4 \mathrm{AaBb} \\
4 \mathrm{Aabb}\end{array}$ & $\begin{array}{l}8 \mathrm{AaBB} \\
8 \mathrm{AaBb}\end{array}$ & $\begin{array}{l}4 \mathrm{AaBB} \\
8 \mathrm{AaBb} \\
4 \mathrm{Aabb}\end{array}$ & $\begin{array}{l}8 \mathrm{AaBb} \\
8 \mathrm{Aabb}\end{array}$ \\
\hline Aabb & $16 \mathrm{AABb}$ & $\begin{array}{l}8 \mathrm{AABb} \\
8 \mathrm{AAbb}\end{array}$ & 16AAbb & $\begin{array}{l}8 \mathrm{AABb} \\
8 \mathrm{AaBb}\end{array}$ & $\begin{array}{l}4 \mathrm{AABb} \\
4 \mathrm{AAbb} \\
4 \mathrm{AaBb} \\
4 \mathrm{Aabb}\end{array}$ & $\begin{array}{l}8 \mathrm{AAbb} \\
8 \mathrm{Aabb}\end{array}$ & $16 \mathrm{AaBb}$ & $\begin{array}{l}8 \mathrm{AaBb} \\
8 \mathrm{Aabb}\end{array}$ & $16 \mathrm{Aabb}$ \\
\hline AaBB & $\begin{array}{l}8 \mathrm{AABB} \\
8 \mathrm{AaBB}\end{array}$ & $\begin{array}{l}4 \mathrm{AABB} \\
4 \mathrm{AABb} \\
4 \mathrm{AaBB} \\
4 \mathrm{AaBb}\end{array}$ & $\begin{array}{l}8 \mathrm{AABb} \\
8 \mathrm{AaBb}\end{array}$ & $\begin{array}{c}4 \mathrm{AABB} \\
8 \mathrm{AaBB} \\
4 \mathrm{aaBB}\end{array}$ & $\begin{array}{l}2 \mathrm{AABB} \\
2 \mathrm{AABb} \\
4 \mathrm{AaBB} \\
4 \mathrm{AaBb} \\
2 \mathrm{aaBB} \\
2 \mathrm{aaBb}\end{array}$ & $\begin{array}{l}4 \mathrm{AABb} \\
8 \mathrm{AaBb} \\
4 \mathrm{aaBb}\end{array}$ & $\begin{array}{l}8 \mathrm{AaBB} \\
8 \mathrm{aaBB}\end{array}$ & $\begin{array}{l}4 \mathrm{AaBB} \\
4 \mathrm{AaBb} \\
4 \mathrm{aaBB} \\
4 \mathrm{aaBb}\end{array}$ & $\begin{array}{c}8 \mathrm{AaBb} \\
8 \mathrm{aaBb}\end{array}$ \\
\hline $\mathrm{AaBb}$ & $\begin{array}{l}4 \mathrm{AABB} \\
4 \mathrm{AABb} \\
4 \mathrm{AaBB} \\
4 \mathrm{AaBb}\end{array}$ & $\begin{array}{l}2 \mathrm{AABB} \\
4 \mathrm{AABb} \\
2 \mathrm{AAbb} \\
2 \mathrm{AaBB} \\
4 \mathrm{AaBb} \\
2 \mathrm{Aabb}\end{array}$ & $\begin{array}{l}4 \mathrm{AABb} \\
4 \mathrm{AAbb} \\
4 \mathrm{AaBb} \\
4 \mathrm{Aabb}\end{array}$ & $\begin{array}{l}2 \mathrm{AABB} \\
2 \mathrm{AABb} \\
4 \mathrm{AaBB} \\
4 \mathrm{AaBb} \\
2 \mathrm{aaBB} \\
2 \mathrm{aaBb}\end{array}$ & $\begin{array}{c}1 \mathrm{AABB} \\
2 \mathrm{AABb} \\
1 \mathrm{AAbb} \\
2 \mathrm{AaBB} \\
4 \mathrm{AaBb} \\
2 \mathrm{Aabb} \\
1 \mathrm{aaBB} \\
2 \mathrm{aaBb} \\
1 \mathrm{aabb}\end{array}$ & $\begin{array}{l}2 \mathrm{AABb} \\
2 \mathrm{AAbb} \\
4 \mathrm{AaBb} \\
4 \mathrm{Aabb} \\
2 \mathrm{aaBb} \\
2 \mathrm{aabb}\end{array}$ & $\begin{array}{l}4 \mathrm{AaBB} \\
4 \mathrm{AaBb} \\
4 \mathrm{aaBB} \\
4 \mathrm{aaBb}\end{array}$ & $\begin{array}{l}2 \mathrm{AaBB} \\
4 \mathrm{AaBb} \\
2 \mathrm{Aabb} \\
2 \mathrm{aaBB} \\
4 \mathrm{aaBb} \\
2 \mathrm{aabb}\end{array}$ & $\begin{array}{l}4 \mathrm{AaBb} \\
4 \mathrm{Aabb} \\
4 \mathrm{aaBb} \\
4 \mathrm{aabb}\end{array}$ \\
\hline Aabb & $\begin{array}{l}8 \mathrm{AABb} \\
8 \mathrm{AaBb}\end{array}$ & $\begin{array}{l}4 \mathrm{AABb} \\
4 \mathrm{AAbb} \\
4 \mathrm{AaBb} \\
4 \mathrm{Aabb}\end{array}$ & $\begin{array}{l}8 \mathrm{AAbb} \\
8 \mathrm{Aabb}\end{array}$ & $\begin{array}{c}4 \mathrm{AABb} \\
8 \mathrm{AaBb} \\
4 \mathrm{aaBb}\end{array}$ & $\begin{array}{l}2 \mathrm{AABb} \\
2 \mathrm{AAbb} \\
4 \mathrm{AaBb} \\
4 \mathrm{Aabb} \\
2 \mathrm{aaBb} \\
2 \mathrm{aabb}\end{array}$ & $\begin{array}{c}\text { 4AAbb } \\
8 \mathrm{Aabb} \\
4 \mathrm{aabb}\end{array}$ & $\begin{array}{l}8 \mathrm{AaBb} \\
8 \mathrm{aaBb}\end{array}$ & $\begin{array}{l}4 \mathrm{AaBb} \\
4 \mathrm{Aabb} \\
4 \mathrm{aaBb} \\
4 \mathrm{aabb}\end{array}$ & $\begin{array}{l}8 \mathrm{Aabb} \\
8 \mathrm{aabb}\end{array}$ \\
\hline $\mathrm{aaBB}$ & 16AaBB & $\begin{array}{l}8 \mathrm{AaBB} \\
8 \mathrm{AaBb}\end{array}$ & $16 \mathrm{AaBb}$ & $\begin{array}{l}8 \mathrm{AaBB} \\
8 \mathrm{aaBB}\end{array}$ & $\begin{array}{l}4 \mathrm{AaBB} \\
4 \mathrm{AaBb} \\
4 \mathrm{aaBB} \\
4 \mathrm{aaBb}\end{array}$ & $\begin{array}{l}8 \mathrm{AaBb} \\
8 \mathrm{aaBb}\end{array}$ & 16аaBB & $\begin{array}{l}8 \mathrm{aaBB} \\
8 \mathrm{aaBb}\end{array}$ & $16 \mathrm{aaBb}$ \\
\hline $\mathrm{aaBb}$ & $\begin{array}{l}8 \mathrm{AaBB} \\
8 \mathrm{AaBb}\end{array}$ & $\begin{array}{l}4 \mathrm{AaBB} \\
8 \mathrm{AaBb} \\
4 \mathrm{Aabb}\end{array}$ & $\begin{array}{l}8 \mathrm{AaBb} \\
8 \mathrm{Aabb}\end{array}$ & $\begin{array}{l}4 \mathrm{AaBB} \\
4 \mathrm{AaBb} \\
4 \mathrm{aaBB} \\
4 \mathrm{aaBb}\end{array}$ & $\begin{array}{l}2 \mathrm{AaBB} \\
4 \mathrm{AaBb} \\
2 \mathrm{Aabb} \\
2 \mathrm{aaBB} \\
4 \mathrm{aaBb} \\
2 \mathrm{aabb}\end{array}$ & $\begin{array}{l}4 \mathrm{AaBb} \\
4 \mathrm{Aabb} \\
4 \mathrm{aaBb} \\
4 \mathrm{aabb}\end{array}$ & $\begin{array}{l}8 \mathrm{aaBB} \\
8 \mathrm{aaBb}\end{array}$ & $\begin{array}{c}4 a a B B \\
8 a a B b \\
4 a a b b\end{array}$ & $\begin{array}{l}8 \mathrm{aaBb} \\
8 \mathrm{aabb}\end{array}$ \\
\hline aabb & $16 \mathrm{AaBb}$ & $\begin{array}{l}8 \mathrm{AaBb} \\
8 \mathrm{Aabb}\end{array}$ & 16Aabb & $\begin{array}{l}8 \mathrm{AaBb} \\
8 \mathrm{aaBb}\end{array}$ & $\begin{array}{l}4 \mathrm{AaBb} \\
4 \mathrm{Aabb} \\
4 \mathrm{aaBb} \\
4 \mathrm{aabb}\end{array}$ & $\begin{array}{l}8 \mathrm{Aabb} \\
8 \mathrm{aabb}\end{array}$ & $16 \mathrm{aaBb}$ & $\begin{array}{l}8 \mathrm{aaBb} \\
8 \mathrm{aabb}\end{array}$ & 16aabb \\
\hline
\end{tabular}

explored here. In the first, it is hypothesized that $\mathrm{AABB}$ survives best $\left(S_{1}=1\right)$ since AA confers a strong attraction to the host, while $\mathrm{BB}$ allows the beetle to avoid feeding in the nonhost that would kill the individual. AABb survives well $\left(S_{2}=0.8\right)$ for the same reasons although Bb, being intermediate, causes some attacks on nonhosts and mortality. AAbb has considerably lower survival $\left(S_{3}=0.5\right)$ due to bb causing nonhost feeding and mortality, but it does allow many AA to find hosts. AaBB $\left(S_{4}=0.9\right)$ can be given higher survival than $\mathrm{AABb}$ because individuals of the former avoid nonhosts that is slightly more important than a specific attraction to hosts. Heterozygous $\mathrm{AaBb}\left(S_{5}=0.6\right)$ has intermediate survival, while Aabb $\left(\mathrm{S}_{6}=0.3\right)$ and aaBb $\left(\mathrm{S}_{8}=0.3\right)$ are of equally low survival. The aaBB $\left(S_{7}=0.4\right)$ has slightly more survival due 


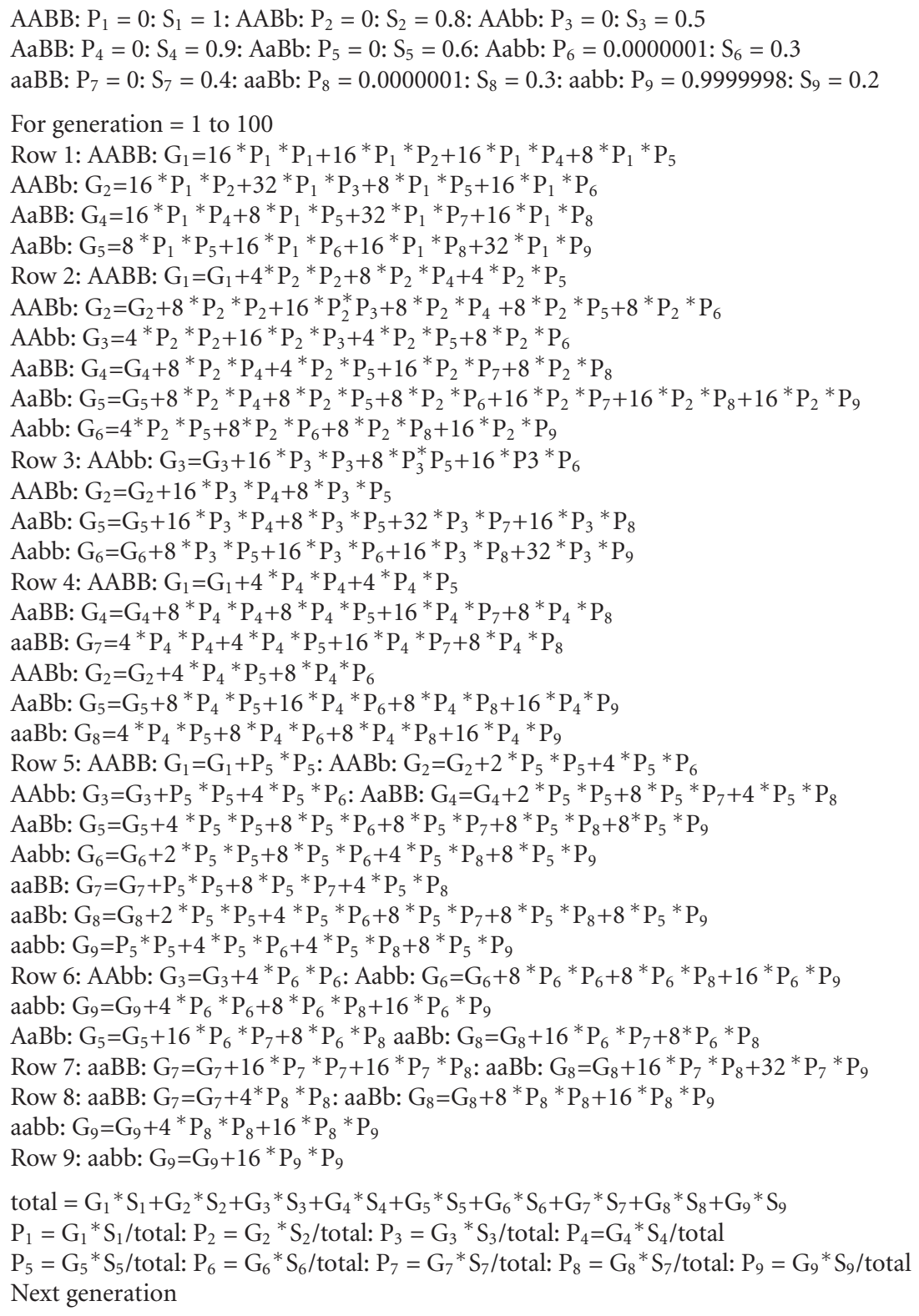

Algorithm 1: General code for algorithms to calculate the proportions $\mathrm{P}_{1}$ to $\mathrm{P}_{9}$ of the nine mated genotypes (AABB to aabb, see Table 1 ) for 200 generations based on initial proportions and nine constant survival factors $S_{1}$ to $S_{9}$ for individuals of these genotypes (an asterisk denotes multiplication).

to the importance of avoiding toxic nonhosts, while the genotype with the least survival would be aabb $\left(S_{9}=0.2\right)$. Thus, the order of survival was $\mathrm{S}_{1}>\mathrm{S}_{4}>\mathrm{S}_{2}>\mathrm{S}_{5}>\mathrm{S}_{3}>\mathrm{S}_{7}>\mathrm{S}_{6}=$ $\mathrm{S}_{8}>\mathrm{S}_{9}$ (Table 2).

In the second example, the initial proportions were the same, and the survival factors were similar: $\mathrm{S}_{1}(1)>\mathrm{S}_{4}(0.8)>$ $\mathrm{S}_{7}(0.7)>\mathrm{S}_{2}(0.6)>\mathrm{S}_{5}(0.5)>\mathrm{S}_{8}(0.4)>\mathrm{S}_{3}(0.3)>\mathrm{S}_{6}(0.2)>$ $S_{9}(0.1)$. However, $S_{7}$ had significantly higher survival, as did $\mathrm{S}_{8}$, than in the first example (Table 2). The justification was that the $\mathrm{BB}$ of aaBB would allow avoidance of toxic nonhosts better than $\mathrm{Bb}$ of $\mathrm{AABb}$, and this advantage outweighs the benefits of a better attraction to hosts (AA versus aa). In the third example, the survival factors were $S_{2}(1)>S_{1}(0.9)>$ $\mathrm{S}_{5}(0.8)>\mathrm{S}_{4}(0.7)>\mathrm{S}_{8}(0.5)>\mathrm{S}_{7}(0.4)=\mathrm{S}_{3}(0.4)>\mathrm{S}_{6}(0.3)>$ $\mathrm{S}_{9}(0.2)$. The rationale for this order was that the $\mathrm{BB}$ gene caused these bark beetles to be somewhat repelled from forests with nonhost trees [39] so these beetles found hosts less often than $\mathrm{Bb}$, thus $\mathrm{Bb}>\mathrm{BB}>\mathrm{bb}$ and $\mathrm{AA}>\mathrm{Aa}>$ aa in survival. In the fourth model (Table 2 ), the order was affected by the preceding rationale for $\mathrm{BB}$, but in addition $\mathrm{AA}$ caused too much attraction and competition, while Aa allowed less attraction to crowded hosts and increased survival [40-43]. 
TABLE 2: Survival factors $\left(S_{1}\right.$ to $\left.S_{9}\right)$ of the nine genotypes used in the five example models.

\begin{tabular}{lcccccccccc}
\hline Example & $\mathrm{S}_{1} \mathrm{AABB}$ & $\mathrm{S}_{2} \mathrm{AABb}$ & $\mathrm{S}_{3} \mathrm{AAbb}$ & $\mathrm{S}_{4}$ AaBB & $\mathrm{S}_{5} \mathrm{AaBb}$ & $\mathrm{S}_{6}$ Aabb & $\mathrm{S}_{7} \mathrm{aaBB}$ & $\mathrm{S}_{8} \mathrm{aaBb}$ & $\mathrm{S}_{9} \mathrm{aabb}$ \\
\hline 1 & 1 & 0.8 & 0.5 & 0.9 & 0.6 & 0.3 & 0.4 & 0.3 & 0.2 \\
2 & 1 & 0.6 & 0.3 & 0.8 & 0.5 & 0.2 & 0.7 & 0.4 & 0.1 \\
3 & 0.9 & 1 & 0.4 & 0.7 & 0.8 & 0.3 & 0.4 & 0.5 & 0.2 \\
4 & 0.8 & 0.9 & 0.4 & 0.4 & 1 & 0.5 & 0.1 & 0.5 & 0.2 \\
5 & 1 & 0.98 & 0.95 & 0.99 & 0.96 & 0.93 & 0.94 & 0.93 & 0.92 \\
\hline
\end{tabular}

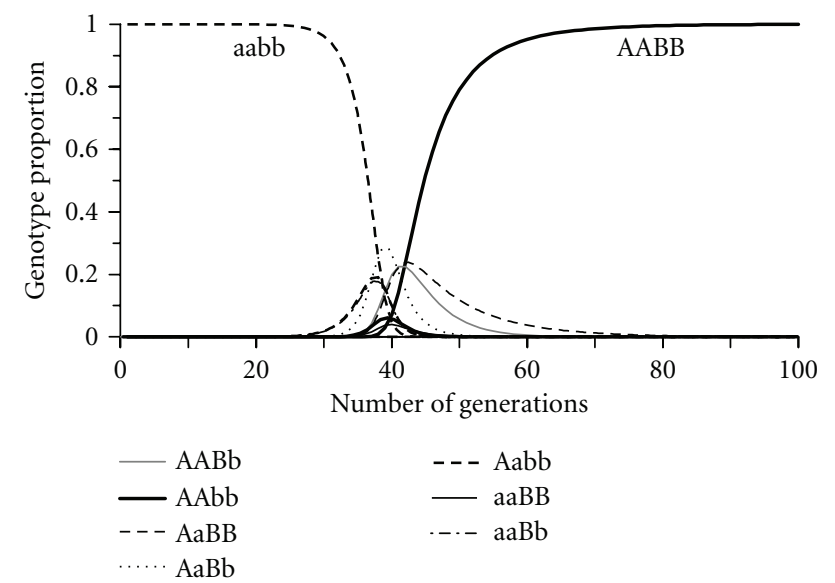

FIGURE 1: Change in proportions of the nine genotypes during 100 generations (example 1) with indiscriminate mating in which there were initially about $10^{7}$ (0.9999998 aabb) individuals and initially one mutation of a to $\mathrm{A}(0.0000001 \mathrm{Aabb})$ and one of $\mathrm{b}$ to $\mathrm{B}$ $(0.0000001 \mathrm{aaBb})$ resulting in $0.9994339 \mathrm{AABB}$ after 100 generations. Relative survival of each genotype was $S_{1}=1$ (AABB), $S_{2}=0.8$ $(\mathrm{AABb}), \mathrm{S}_{3}=0.5(\mathrm{AAbb}), \mathrm{S}_{4}=0.9(\mathrm{AaBB}), \mathrm{S}_{5}=0.6(\mathrm{AaBb}), \mathrm{S}_{6}=0.3$ $(\mathrm{Aabb}), \mathrm{S}_{7}=0.4(\mathrm{aaBB}), \mathrm{S}_{8}=0.3(\mathrm{aaBb})$, and $\mathrm{S}_{9}=0.2(\mathrm{aabb})$.

Thus, Aa $>$ AA $>$ aa, giving an order of $S_{5}(1)>S_{2}(0.9)=S_{4}$ $(0.9)>S_{1}(0.8)>S_{8}(0.5)=S_{6}(0.5)>S_{3}(0.4)>S_{9}(0.2)>S_{7}$ $(0.1)$.

The effect of smaller relative differences in the survival factors was tested in a fifth example where the first model's factors (Table 2) were altered $S_{1}(1)>S_{4}(0.99)>S_{2}(0.98)>$ $S_{5}(0.96)>S_{3}(0.95)>S_{7}(0.94)>S_{6}(0.93)=S_{8}(0.93)>S_{9}$ (0.92), and the number of generations was noted at which $P_{1}$ $(\mathrm{AABB})>0.01$ or $\mathrm{P}_{9}(\mathrm{aabb})<0.99$. The model (Algorithm 1) was programmed in QuickBASIC 4.5 (Microsoft Corp., Redmond, WA, USA) with results graphed using PostScript 2.0 language (Adobe Systems Inc., San Jose, CA, USA). The model was also implemented in Java 6.0 code (Oracle, Redwood City, CA, USA) for general demonstration on the Internet with a web browser (http://www.chemical-ecology .net/java2/aabb.htm).

\section{Results}

The predominate initial genotype aabb, with no significant attraction to host volatiles and no avoidance of nonhost volatiles, appears stable for almost 30 generations before plummeting rapidly to near zero by generation 42 (Figure 1).

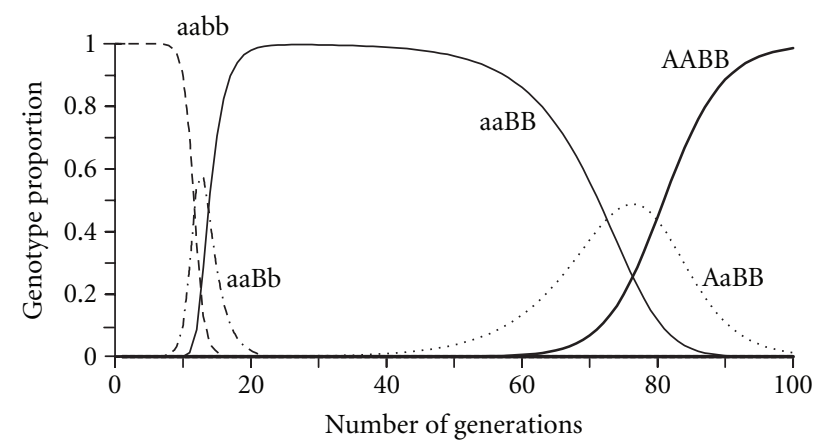

FIGURE 2: Change in proportions of the nine genotypes during 100 generations (example 2) with indiscriminate mating in which there were initially about $10^{7}$ (0.9999998 aabb) individuals and initially one mutation of a to A (0.0000001 Aabb) and one of b to B $(0.0000001 \mathrm{aaBb})$ resulting in $0.986431 \mathrm{AABB}$ (four genotypes never achieved any significant proportion). Relative survival of each genotype was $S_{1}=1(\mathrm{AABB}), \mathrm{S}_{2}=0.6(\mathrm{AABb}), \mathrm{S}_{3}=0.3(\mathrm{AAbb}), \mathrm{S}_{4}=$ $0.8(\mathrm{AaBB}), \mathrm{S}_{5}=0.5(\mathrm{AaBb}), \mathrm{S}_{6}=0.2(\mathrm{Aabb}), \mathrm{S}_{7}=0.7(\mathrm{aaBB}), \mathrm{S}_{8}=$ $0.4(\mathrm{aaBb})$, and $\mathrm{S}_{9}=0.1(\mathrm{aabb})$.

Concomitantly, the dominant genotype AABB logistically grows to 1.0 from generations 38 to 60 and reaches 0.9994 by generation 100 . The other seven genotypes rise and fall in approximate normal curves with some skews during generations 25 to 80 (Figure 1). It is apparent that AABB will fixate to $100 \%$ eventually.

In the second example, the initial aabb genotype also declines precipitously after near constancy for about 10 generations and approaches zero by generation 15 (Figure 2). However, AABB does not increase above zero for a considerable time until about generation 60 whereupon $\mathrm{AABB}$ rises logistically to 0.9864 by 100 generations. It is again clear that AABB fixates. For a number of generations aaBB rises after generation 10 and approaches fixation by generation 22 but then declines gradually until about generation 60 when the genotype then falls to zero (the same period when $\mathrm{AABB}$ increases). Only aaBb and $\mathrm{AaBB}$ genotypes rise and fall (as Gaussian-like curves) substantially during the fall of aabb and rise of $\mathrm{AABB}$, respectively (Figure 2). The fixation occurs on a time scale that is similar to that found for pesticide resistance in insects and nematodes $[37,38,44]$ and indicates that the survival factors chosen here are reasonable for strong selection.

In example 3 (Table 2), the initial aabb is again stable for about 12 generations and then falls when aaBb begins to increase (Figure 3). Other genotypes, aaBB, $\mathrm{AaBb}$, and $\mathrm{Aabb}$ 


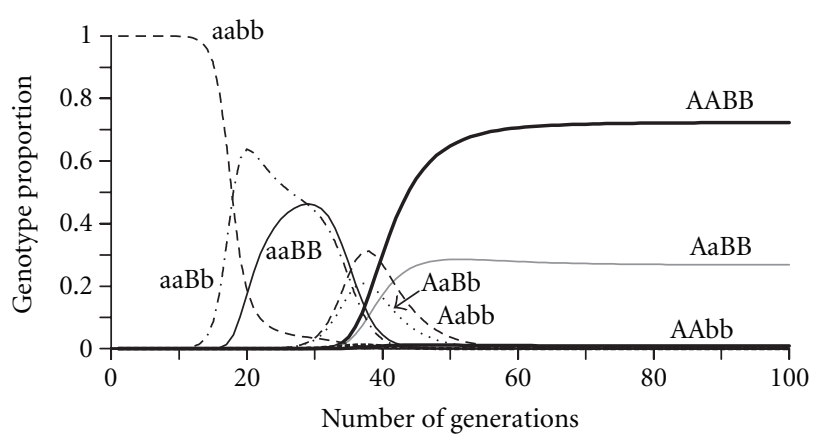

FIGURE 3: Change in proportions of the nine genotypes during 100 generations (example 3 ) with indiscriminate mating in which there were initially about $10^{7}(0.9999998 \mathrm{aabb})$ individuals and initially one mutation of a to A $(0.0000001 \mathrm{Aabb})$ and one of $\mathrm{b}$ to $\mathrm{B}$ $(0.0000001 \mathrm{aaBb})$ resulting in $0.7230 \mathrm{AABB}, 0.2681 \mathrm{AABb}$, and 0.09 $\mathrm{AAbb}(\mathrm{AABb}$ never achieved any significant proportion). Relative survival of each genotype was $S_{1}=0.9(\mathrm{AABB}), \mathrm{S}_{2}=1(\mathrm{AABb}), \mathrm{S}_{3}=$ $0.4(\mathrm{AAbb}), \mathrm{S}_{4}=0.7(\mathrm{AaBB}), \mathrm{S}_{5}=0.8(\mathrm{AaBb}), \mathrm{S}_{6}=0.3(\mathrm{Aabb}), \mathrm{S}_{7}=$ $0.4(\mathrm{aaBB}), \mathrm{S}_{8}=0.5(\mathrm{aaBb})$, and $\mathrm{S}_{9}=0.2(\mathrm{aabb})$.

rise, and fall during the rise of $\mathrm{AABB}$ and $\mathrm{AaBB}$ around generation 33. It is remarkable that $A A B B$ appears to reach equilibrium at 0.7230 , as does $\mathrm{AABb}$ at 0.2681 , accounting for most of the population's genotypes at 100 generations. AAbb reaches a low level of equilibrium at 0.0895 (9 percent). Running the model to 1000 generations did not appreciably change these results $(\mathrm{AABB}=0.7232, \mathrm{AABb}=0.2679$, and $\mathrm{AAbb}=0.0893)$.

In example 4 (Table 2), the initial aabb genotype is stable until about generation 12 and falls rapidly to near 0 by generation 22 while three genotypes (Aabb, aaBb, and AAbb) rise after generation 10 and then decline around generation 20 , reaching near 0 levels asymptotically (Figure 4 ). A few generations before 20, four genotypes rise, with $\mathrm{AaBb}$ falling gradually but then reaching a constant equilibrium of about 0.0779. The dominant genotype AABB appears to rise logarithmically to a stable equilibrium that was 0.5037 by generation 100. Similarly, AABb and AaBB rose and then fell slightly to stable equilibriums at 0.2374 and 0.1614 , respectively (Figure 4). Running the model to 1000 generations did not change the results $(\mathrm{AABB}=0.5039, \mathrm{AABb}=0.2373, \mathrm{AaBB}=$ $0.1614, \mathrm{AaBb}=0.0779, \mathrm{AAbb}=0.0110)$. The other genotypes, Aabb, aaBB, aaBb, and aabb, also became stable but below 0.0050 proportion. In example 5 (Table 2 ), the survival parameters were compressed but related to example 1 . In this case, the genotypic frequencies were identical but spread out over more generations (not shown). In example 1, the initial population of aabb began to decline significantly when the proportion fell below 0.99 on generation 27 while the $\mathrm{AABB}$ proportion began to significantly increase above 0.01 on generation 39. Using the compressed survival factors that caused less selection in example 5, aabb fell below 0.99 on generation 1001, and AABB rose above 0.01 on generation 1394.

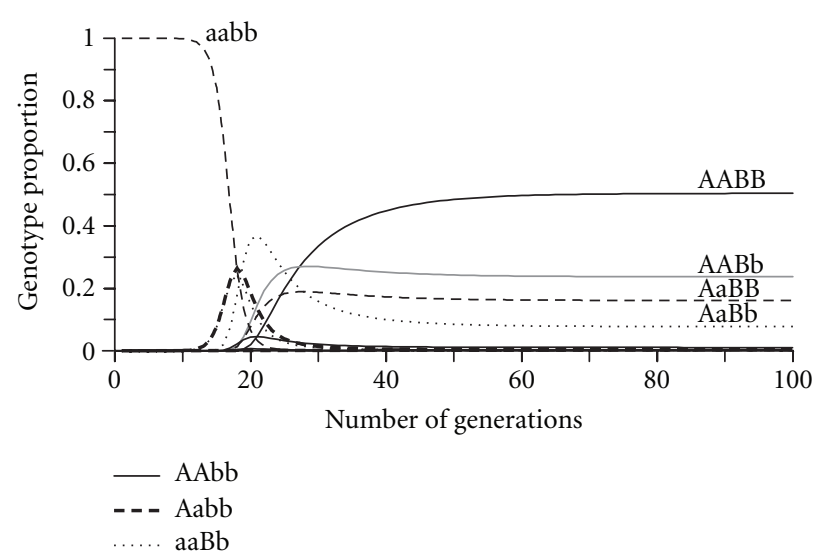

Figure 4: Change in proportions of the nine genotypes during 100 generations (example 4) with indiscriminate mating in which there were initially about $10^{7}(0.9999998$ aabb) individuals and initially one mutation of a to A (0.0000001 Aabb) and one of b to $\mathrm{B}(0.0000001 \mathrm{aaBb})$ resulting in $0.5037 \mathrm{AABB}, 0.2376 \mathrm{AABb}$, and $0.1614 \mathrm{AaBB}$. Relative survival of each genotype was $S_{1}=0.8$ $(\mathrm{AABB}), \mathrm{S}_{2}=0.9(\mathrm{AABb}), \mathrm{S}_{3}=0.4(\mathrm{AAbb}), \mathrm{S}_{4}=0.9(\mathrm{AaBB}), \mathrm{S}_{5}=1$ $(\mathrm{AaBb}), \mathrm{S}_{6}=0.5(\mathrm{Aabb}), \mathrm{S}_{7}=0.1(\mathrm{aaBB}), \mathrm{S}_{8}=0.5(\mathrm{aaBb})$, and $S_{9}=$ 0.2 (aabb).

\section{Discussion}

Bark beetles that are termed "aggressive" are among the treekilling pest species thought to find trees in either of two ways. The first is through a nondirected flight and landing on trees at random whereupon the beetle, a male if in tribe Ipini (e.g., Pityogenes or Ips) or a female if in tribe Tomicini (e.g., Dendroctonus or Tomicus), must determine whether the tree is its host and probably whether the tree is acceptable $[24,45]$. A beetle that lands on a tree and attempts to enter by boring through the outer bark is termed a "pioneer," especially if there are few others present. Pioneers were presumed to encounter significant host resistance and resin when attacking compared to later arrivals ("joiners") when the tree has succumbed $[21,22,24,46]$. The hypothesis was that since pioneers must attack the tree and survive to produce pheromone before the rest of the population can exploit the resource, pioneers must be the largest and most vigorous of the population. Byers [24] questioned this paradigm since an individual would undertake a pioneer strategy only if no pheromone was encountered during the dispersal, or after leaving unsuitable colonization areas $[41,43]$, so that eventually its fat reserves became low [47]. In this "desperate" state, the beetle attempts to bore into any tree and may fortuitously find a tree of low resistance. Thus, smaller beetles that have suffered severe larval competition, or beetles regardless of size that have used up their fat reserves in flight, are hypothesized to be the pioneers.

The second way a beetle finds a host is by orienting to aggregation pheromone. It is evident from host finding models using EAR (effective attraction radius), representing trees and hosts under colonization, that the vast majority of beetles find hosts by orientation to aggregation pheromone [23]. This still means that many beetles perish as pioneers or 
simply in the search for hosts; however, most find their host by means of aggregation pheromone. As mentioned earlier, some species in the genus Tomicus (e.g., T. piniperda) appear not to use a long-range aggregation pheromone but rely instead on volatile monoterpenes predominant in the hosts of their region (in Sweden: $\alpha$-pinene, 3-carene, and terpinolene) [27-29]. P. bidentatus has a strong aggregation pheromone, and thus most individuals would seem to find hosts by the use of these semiochemicals. However, fresh hosts, even with synthetic aggregation pheromone, are repellent, while aged logs in the field may be attractive or located through a random landing process. Avoidance of nonhost volatiles has evolved in a number of bark beetles as stated earlier including $P$. bidentatus. All bark beetles colonize a thin layer (often only $2-3 \mathrm{~mm}$ ) of cambium/phloem that causes both intraspecific and interspecific competition for food resources [48]. Thus, bark beetles have evolved avoidance of verbenone and aggregation pheromone in order to reduce competition [21, 22, 28, 29, 40, 43, 49].

The attraction to host odors, aggregation pheromone, and avoidance of volatiles indicative of crowding can be implemented in the survival factors of the hypothetical (A, a) gene. The avoidance of nonhost odors, both at the tree and forest stand level, and semiochemicals from unsuitable hosts was incorporated in the assumptions about the survival factors conferred by the $(\mathrm{B}, \mathrm{b})$ gene. The relative magnitudes of the survival factors used in the five examples were ecologically reasonable, but many other relative rankings are possible. The speed of evolution can be greatly affected based on the survival factors that represent selection pressures. In example 1 (Figure 1), 39 generations transpired before the first sign of an increase in $\mathrm{AABB}$. It took until generation 45 for $50 \%$ of the populations to become AABB. In example 5 with the same order but less difference in relative survival factors, it took 1394 generations before AABB began to increase, and this genotype did not reach $50 \%$ until generation 1498 (about 104 generations to increase to 50\%).

These results demonstrate that eventually there is a relatively rapid change in the genotype frequencies with fixation of the dominant alleles that are the most beneficial. This evolution is analogous to a mutation for resistance to an insecticide [36-38, 44, 50-52]. It supports why resistance may remain hidden phenotypically for many years ( 1 generation per year) before suddenly appearing to become widespread. Resistance to insecticides from a mutation that commonly shows up in several to tens of years would have survival factors similar to those used in the present study. According to example 5, resistance in a pest insect could remain hidden for hundreds of years before becoming established. In examples 3 and 4 , in which the beneficial genes are heterozygous (Figures 3 and 4), it is evident that phenotypic changes can also take many generations before intermediate gene frequencies result that are stable thereafter. On the other hand, if the population already has an allele that is common that confers insecticide resistance, then there can be an immediate and rapid change favoring this gene, which fixates or reaches equilibriums as in the examples. The population genetic models show how only two loci with two alleles can result in complex genotypic frequencies. More genes are probably involved in both choosing a host and avoiding nonhosts in P. bidentatus, which makes the models, undoubtedly, exceedingly complex.

\section{References}

[1] B. Lekander, B. Bejer Peterson, F. Kangas, and A. Bakke, "The distribution of bark beetles in the Nordic countries," Acta Entomologia Fennica, vol. 32, pp. 1-36, 1977.

[2] E. R. Hoebeke, "Pityogenes bidentatus (Herbst), a European bark beetle new to North America (Coleoptera: Scolytidae)," Journal of New York Entomological Society, vol. 97, no. 3, pp. 305-308, 1989.

[3] V. E. J. Baader, "Pityogenes spp. (Col., Scolytidae): Utersuchungen über verhaltenssteuernde Duftstoffe und deren Anwendung im Waldschutz," Journal of Applied Entomology, vol. 107, no. 1, pp. 1-31, 1989.

[4] W. Francke, J. Bartels, H. Meyer et al., "Semiochemicals from bark beetles: new results, remarks, and reflections," Journal of Chemical Ecology, vol. 21, no. 7, pp. 1043-1063, 1995.

[5] J. A. Byers, Q. H. Zhang, and G. Birgersson, "Strategies of a bark beetle, Pityogenes bidentatus, in an olfactory landscape," Naturwissenschaften, vol. 87, no. 11, pp. 503-507, 2000.

[6] J. A. Byers, Q. H. Zhang, and G. Birgersson, "Avoidance of nonhost plants by a bark beetle, Pityogenes bidentatus, in a forest of odors," Naturwissenschaften, vol. 91, no. 5, pp. 215219, 2004.

[7] A. M. El-Sayed and J. A. Byers, "Inhibitory effect of monoterpenes on response of Pityogenes bidentatus to aggregation pheromone released by piezoelectric sprayer for precision release of semiochemicals," Journal of Chemical Ecology, vol. 26, no. 8, pp. 1795-1809, 2000.

[8] J. A. Byers, F. Schlyter, G. Birgersson, and W. Francke, "Emyrcenol in Ips duplicatus: an aggregation pheromone component new for bark beetles," Experientia, vol. 46, no. 11-12, pp. 1209-1211, 1990.

[9] J. C. Dickens, R. F. Billings, and T. L. Payne, "Green leaf volatiles interrupt aggregation pheromone response in bark beetles infesting southern pines," Experientia, vol. 48, no. 5, pp. 523524, 1992.

[10] L. M. Schroeder, "Olfactory recognition of nonhosts aspen and birch by conifer bark beetles Tomicus piniperda and Hylurgops palliatus," Journal of Chemical Ecology, vol. 18, no. 9, pp. 15831593, 1992.

[11] I. M. Wilson, J. H. Borden, R. Gries, and G. Gries, "Green leaf volatiles as antiaggregants for the mountain pine beetle, Dendroctonus ponderosae Hopkins (Coleoptera: Scolytidae)," Journal of Chemical Ecology, vol. 22, no. 10, pp. 1861-1875, 1996.

[12] A. Guerrero, J. Feixas, J. Pajares, L. J. Wadhams, J. A. Pickett, and C. M. Woodcock, "Semiochemically induced inhibition of behaviour of Tomicus destruens (Woll.) (Coleoptera: Scolytidae)," Naturwissenschaften, vol. 84, no. 4, pp. 155-157, 1997.

[13] J. H. Borden, L. J. Chong, A. Savoie, and I. M. Wilson, "Responses to green leaf volatiles in two biogeoclimatic zones by striped ambrosia beetle, Trypodendron lineatum," Journal of Chemical Ecology, vol. 23, no. 11, pp. 2479-2491, 1997.

[14] J. H. Borden, I. M. Wilson, R. Gries, L. J. Chong, H. D. Pierce, and G. Gries Jr., "Volatiles from the bark of trembling aspen, Populus tremuloides Michx. (Salicaceae) disrupt secondary attraction by the mountain pine beetle, Dendroctonus pondevosae Hopkins (Coleoptera: Scolytidae)," Chemoecology, vol. 8, no. 2, pp. 69-75, 1998. 
[15] E. K. Deglow and J. H. Borden, "Green leaf volatiles disrupt and enhance response to aggregation pheromones by the ambrosia beetle, Gnathotrichus sulcatus (Coleoptera: Scolytidae)," Canadian Journal of Forest Research, vol. 28, no. 11, pp. 1697-1705, 1998.

[16] J. A. Byers, Q. H. Zhang, F. Schlyter, and G. Birgersson, "Volatiles from nonhost birch trees inhibit pheromone response in spruce bark beetles," Naturwissenschaften, vol. 85, no. 11, pp. 557-561, 1998.

[17] Q. H. E. Zhang, G. Birgersson, J. Zhu, C. Löfstedt, J. Löfqvist, and F. Schlyter, "Leaf volatiles from nonhost deciduous trees: variation by tree species, season and temperature, and electrophysiological activity in Ips typographus," Journal of Chemical Ecology, vol. 25, no. 8, pp. 1923-1943, 1999.

[18] Q. H. Zhang, F. Schlyter, and P. Anderson, "Green leaf volatiles interrupt pheromone response of spruce bark beetle, Ips typographus," Journal of Chemical Ecology, vol. 25, no. 12, pp. 2847-2861, 1999.

[19] D. P. W. Huber, R. Gries, J. H. Borden, and H. D. Pierce, "Two pheromones of coniferophagous bark beetles found in the bark of nonhost angiosperms," Journal of Chemical Ecology, vol. 25, no. 4, pp. 805-816, 1999.

[20] T. M. Poland and R. A. Haack, "Pine shoot beetle, Tomicus piniperda (Col., Scolytidae), responses to common green leaf volatiles," Journal of Applied Entomology, vol. 124, no. 2, pp. 63-69, 2000.

[21] J. A. Byers, "Chemical ecology of bark beetles," Experientia, vol. 45, no. 3, pp. 271-283, 1989.

[22] J. A. Byers, "Host tree chemistry affecting colonization in bark beetles," in Chemical Ecology of Insects 2, R. T. Cardé and W. J. Bell, Eds., pp. 154-213, Chapman and Hall, New York, NY, USA, 1995.

[23] J. A. Byers, "An encounter rate model of bark beetle populations searching at random for susceptible host trees," Ecological Modelling, vol. 91, no. 1-3, pp. 57-66, 1996.

[24] J. A. Byers, "Effects of attraction radius and flight paths on catch of scolytid beetles dispersing outward through rings of pheromone traps," Journal of Chemical Ecology, vol. 25, no. 5, pp. 985-1005, 1999.

[25] J. A. Byers and G. Birgersson, "Pheromone production in a bark beetle independent of myrcene precursor in host pine species," Naturwissenschaften, vol. 77, no. 8, pp. 385-387, 1990.

[26] V. Thoss and J. A. Byers, "Monoterpene chemodiversity of ponderosa pine in relation to herbivory and bark beetle colonization," Chemoecology, vol. 16, no. 1, pp. 51-58, 2006.

[27] J. A. Byers, B. S. Lanne, J. Löfqvist, F. Schlyter, and G. Bergström, "Olfactory recognition of host-tree susceptibility by pine shoot beetles," Naturwissenschaften, vol. 72, no. 6, pp. 324-326, 1985.

[28] J. A. Byers, B. S. Lanne, and J. Löfqvist, "Host tree unsuitability recognized by pine shoot beetles in flight," Experientia, vol. 45, no. 5, pp. 489-492, 1989.

[29] B. S. Lanne, F. Schlyter, J. A. Byers et al., "Differences in attraction to semiochemicals present in sympatric pine shoot beetles, Tomicus minor and T. piniperda," Journal of Chemical Ecology, vol. 13, no. 5, pp. 1045-1067, 1987.

[30] J. A. Byers, "Attraction of bark beetles, Tomicus piniperda, Hylurgops palliatus, and Trypodendron domesticum and other insects to short-chain alcohols and monoterpenes," Journal of Chemical Ecology, vol. 18, no. 12, pp. 2385-2402, 1992.

[31] W. D. Bedard, P. E. Tilden, D. L. Wood, R. M. Silverstein, R. G. Brownlee, and J. O. Rodin, "Western pine beetle: field response to its sex pheromone and a synergistic host terpene, myrcene," Science, vol. 164, no. 3885, pp. 1284-1285, 1969.

[32] R. A. Werner, "Response of the beetle, Ips grandicollis, to combinations of host and insect produced attractants," Journal of Insect Physiology, vol. 18, no. 7, pp. 1403-1412, 1972.

[33] J. A. Rudinsky, M. M. Furniss, L. N. Kline, and R. F. Schmitz, "Attraction and repression of Dendroctonus pseudotsugae (Coleoptera: Scolytidae) by three synthetic pheromones in traps in Oregon and Idaho," Canadian Entomologist, vol. 104, no. 6, pp. 815-822, 1972.

[34] J. A. Byers, G. Birgersson, J. Löfqvist, and G. Bergström, “Synergistic pheromones and monoterpenes enable aggregation and host recognition by a bark beetle," Naturwissenschaften, vol. 75, no. 3, pp. 153-155, 1988.

[35] J. Roughgarden, "Population genetics with multiple loci," in Theory of Population Genetics and Evolutionary Ecology: An Introduction, J. Roughgarden, Ed., pp. 111-133, MacMillan, New York, NY, USA, 1979.

[36] D. W. Onstad and C. A. Guse, "Concepts and complexities of population genetics," in Insect Resistance Management: Biology, Economics, and Prediction, D. W. Onstad, Ed., pp. 69-88, Academic Press, London, UK, 2008.

[37] J. Mallet, "The evolution of insecticide resistance: have the insects won?" Trends in Ecology and Evolution, vol. 4, no. 11, pp. 336-340, 1989.

[38] P. C. Lopes, É. Sucena, M. E. Santos, and S. Magalhães, "Rapid experimental evolution of pesticide resistance in C. elegans entails no costs and affects the mating system," PLoS One, vol. 3, no. 11, Article ID e3741, 2008.

[39] Q. H. Zhang and F. Schlyter, "Olfactory recognition and behavioural avoidance of angiosperm nonhost volatiles by conifer-inhabiting bark beetles," Agricultural and Forest Entomology, vol. 6, no. 1, pp. 1-19, 2004.

[40] J. A. Byers and D. L. Wood, "Interspecific inhibition of the response of the bark beetles, Dendroctonus brevicomis and Ips paraconfusus, to their pheromones in the field," Journal of Chemical Ecology, vol. 6, no. 1, pp. 149-164, 1980.

[41] J. A. Byers, "Sex-specific responses to aggregation pheromone: regulation of colonization density in the bark beetle Ips paraconfusus," Journal of Chemical Ecology, vol. 9, no. 1, pp. 129142, 1983.

[42] J. A. Byers, "Nearest neighbor analysis and simulation of distribution patterns indicates an attack spacing mechanism in the bark beetle, Ips typographus (Coleoptera: Scolytidae)," Environmental Entomology, vol. 13, pp. 1191-1200, 1984.

[43] F. Schlyter, J. A. Byers, and J. Löfqvist, "Attraction to pheromone sources of different quantity, quality, and spacing: density-regulation mechanisms in bark beetle Ips typographus," Journal of Chemical Ecology, vol. 13, no. 6, pp. 1503-1523, 1987.

[44] F. R. Groeters and B. E. Tabashnik, "Roles of selection intensity, major genes, and minor genes in evolution of insecticide resistance," Journal of Economic Entomology, vol. 93, no. 6, pp. 1580-1587, 2000.

[45] H. A. Moeck, D. L. Wood, and K. Q. Lindahl, "Host selection behavior of bark beetles (Coleoptera: Scolytidae) attacking Pinus ponderosa, with special emphasis on the western pine beetle, Dendroctonus brevicomis," Journal of Chemical Ecology, vol. 7, no. 1, pp. 49-83, 1981.

[46] A. A. Berryman, "Dynamics of bark beetle populations: towards a general productivity model," Environmental Entomology, vol. 3, no. 4, pp. 579-585, 1974.

[47] P. F. Botterweg, "Dispersal and flight behaviour of the spruce bark beetle Ips typographus in relation to sex, size and fat 
content," Zeitschrift für Angewandte Entomologie, vol. 94, no. 1-5, pp. 466-489, 1982.

[48] D. M. Light, M. C. Birch, and T. D. Paine, "Laboratory study of intraspecific and interspecific competition within and between two sympatric bark beetle species, Ips pini and Ips paraconfusus," Zeitschrift für Angewandte Entomologie, vol. 96, no. 2, pp. 233-241, 1983.

[49] J. A. Byers, "Avoidance of competition by spruce bark beetles, Ips typographus and Pityogenes chalcographus," Experientia, vol. 49, no. 3, pp. 272-275, 1993.

[50] P. J. Daborn, J. L. Yen, M. R. Bogwitz et al., "A single P450 allele associated with insecticide resistance in Drosophila," Science, vol. 297, no. 5590, pp. 2253-2256, 2002.

[51] P. Labbé, C. Berticat, A. Berthomieu et al., "Forty years of erratic insecticide resistance evolution in the mosquito Culex pipiens," PLoS Genetics, vol. 3, no. 11, pp. 2190-2199, 2007.

[52] E. Gazave, C. Chevillon, T. Lenormand, M. Marquine, and M. Raymond, "Dissecting the cost of insecticide resistance genes during the overwintering period of the mosquito Culex pipiens," Heredity, vol. 87, no. 4, pp. 441-448, 2001. 

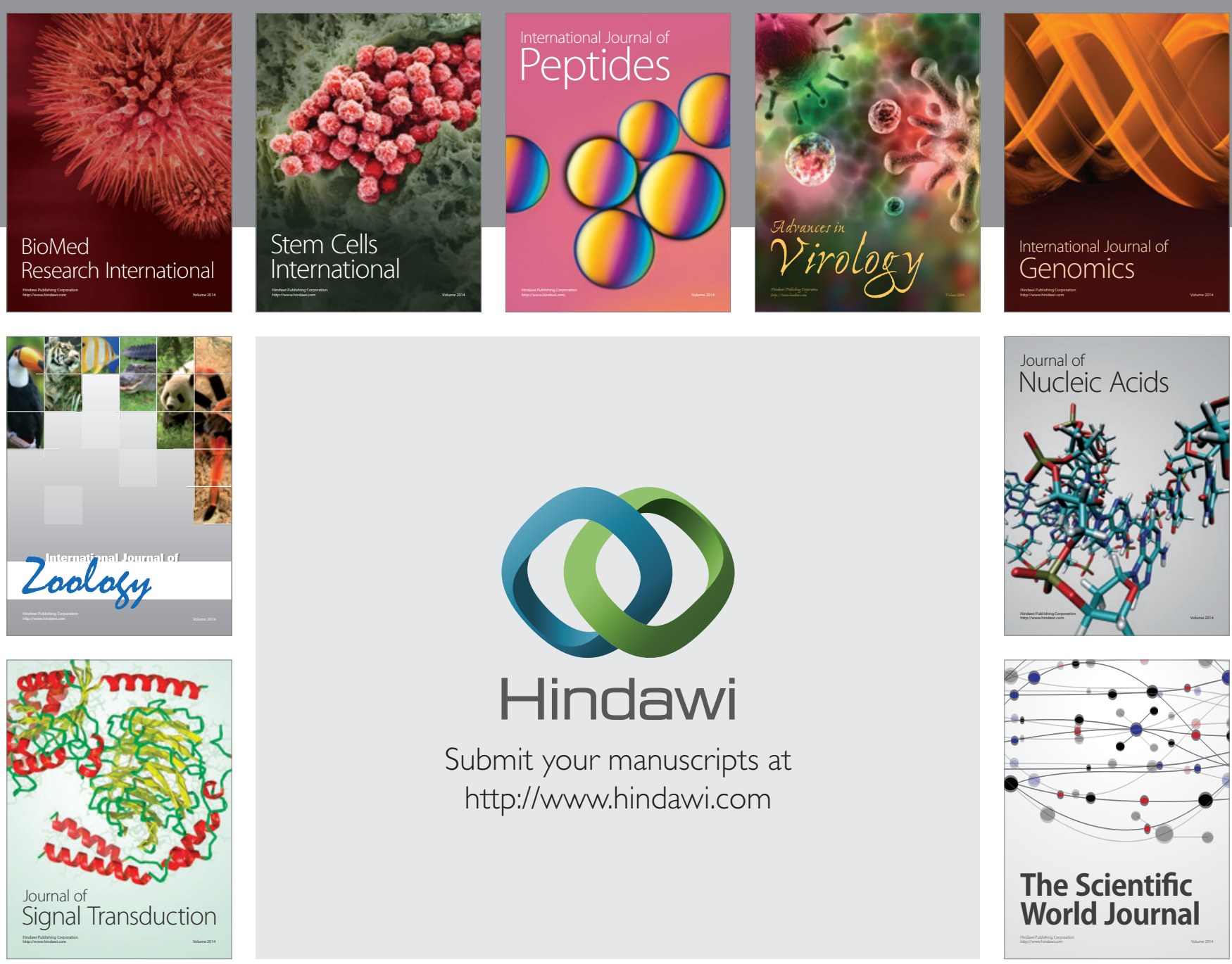

Submit your manuscripts at

http://www.hindawi.com
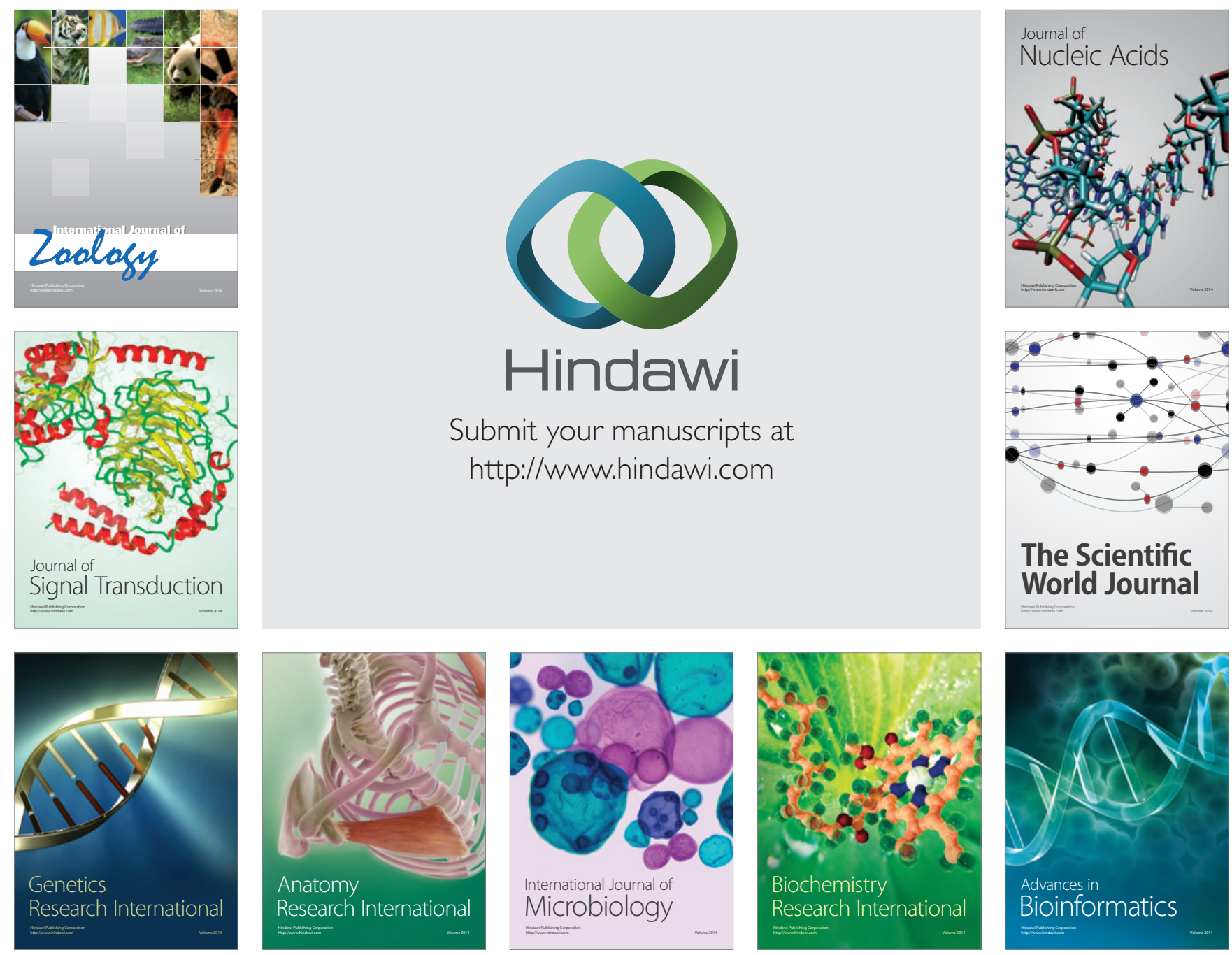

The Scientific World Journal
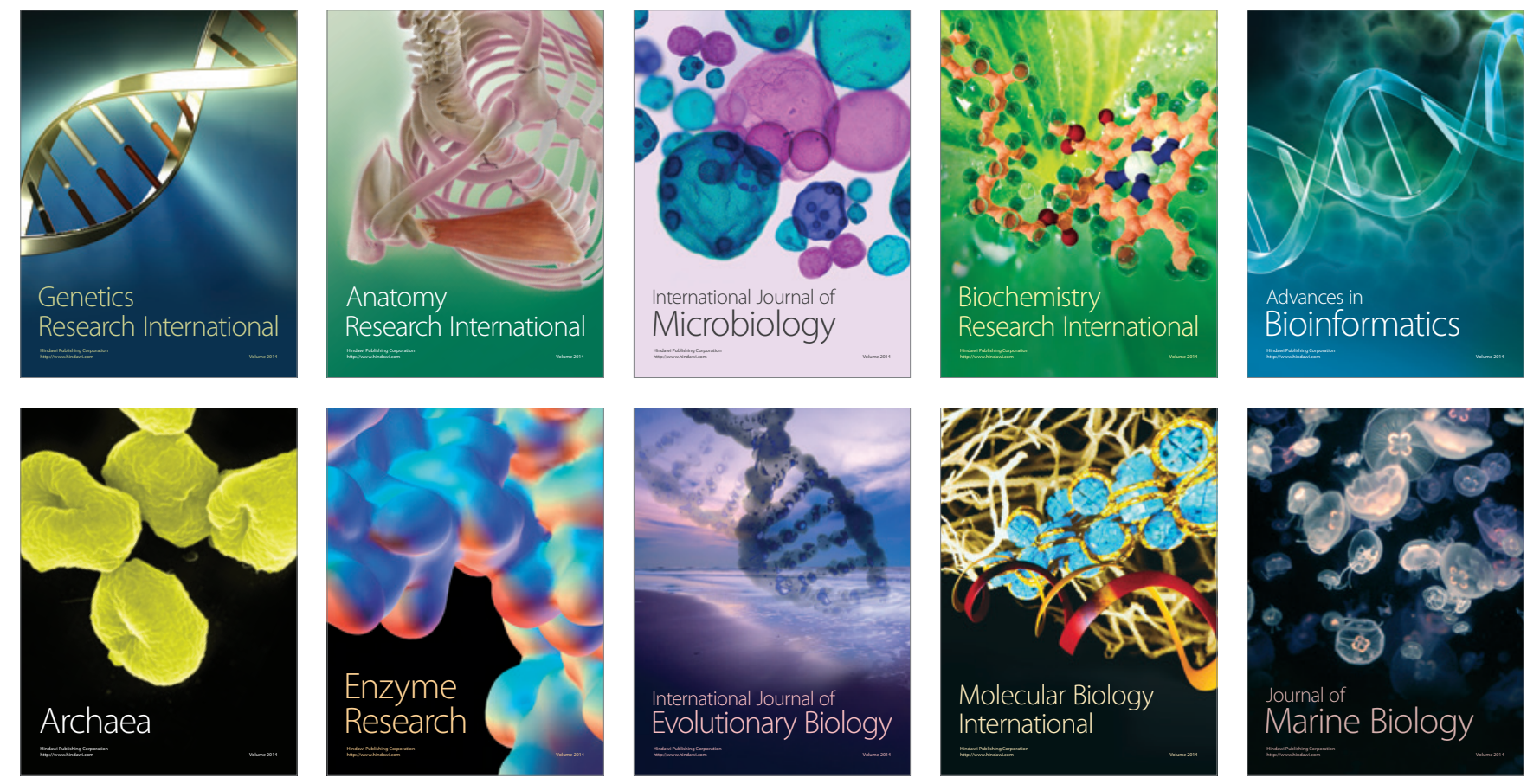